Research Article

\title{
Transient Flow of Jeffrey Fluid over a Permeable Wall
}

\author{
Sana Bajwa, ${ }^{1}$ Saif Ullah $\mathbb{D},{ }^{1}$ Ilyas Khan $\mathbb{D}^{2},{ }^{2}$ and Md. Fayz-Al-Asad $\mathbb{D}^{3}$ \\ ${ }^{1}$ Department of Mathematics, Government College University, Lahore 54000, Pakistan \\ ${ }^{2}$ Department of Mathematics, College of Science Al-Zulf, Majmaah University, Al-Majmaah 11952, Saudi Arabia \\ ${ }^{3}$ Department of Mathematics, Bangladesh University of Engineering and Technology (BUET), Dhaka 1000, Bangladesh
}

Correspondence should be addressed to Ilyas Khan; i.said@mu.edu.sa and Md. Fayz-Al-Asad; fayzmath.buet@gmail.com

Received 5 April 2021; Revised 6 July 2021; Accepted 20 August 2021; Published 9 September 2021

Academic Editor: Mohammad Yaghoub Abdollahzadeh Jamalabadi

Copyright (C) 2021 Sana Bajwa et al. This is an open access article distributed under the Creative Commons Attribution License, which permits unrestricted use, distribution, and reproduction in any medium, provided the original work is properly cited.

\begin{abstract}
Transient incompressible flows of Jeffrey fluids over a permeable, flat, and infinite plate have been investigated. The plate motion is an oscillatory translation along the $x$-axis. Using the Laplace transform and perturbation method, the analytical solution for the velocity field in the transform domain has been obtained. The velocity field in the real domain has been determined by using the numerical Stehfest's algorithm for the Laplace transform inversion. To have a validation of the obtained solution, we have determined the analytical solution for the flow without transpiration. It was found that when the transpiration parameter approaches zero, the solution for the flow with transpiration tends to the solution corresponding to the case without perspiration. The influence of the system parameters on fluid motion has been investigated by numerical simulations and graphical illustrations prepared with the software package Mathcad.
\end{abstract}

\section{Introduction}

The investigation of fluids such as non-Newtonian has currently increased extraordinary attention of the engineers, analysts, and researchers. Such inspiration of scientists is because of different usages of non-Newtonian fluids in innovation and mechanical territories. Not at all like the viscous materials, cannot the non-Newtonian fluids be clarified by utilizing Navier-Stokes hypothesis. A solitary constitutive relationship cannot depict the attributes of non-Newtonian fluids. The specifics of non-Newtonian fluids are particular than the facts of viscous materials. As compared with the differential equations of viscous material, the order of differential equations of non-Newtonian fluids is greater. There are countless types of non-Newtonian fluids by means of differing properties. These fluids have been grouped into three classifications recognized as the differential, integral, and rate type. The most widely recognized and least difficult type of non-Newtonian fluids is Jeffrey fluid. Such fluid has time-derivative rather than convected derivative. Trepathi et al. studied MHD flow in a cylindrical tube of Jeffrey fluid [1]. Das examined impacts of heat transfer and slip on MHD (magnetohydro dynamic) peristaltic flow of Jeffrey fluid [2]. In the occurrence of thermal jump, the variable thermal conductivity of Jeffrey fluid has been considered by Hamad et al. [3]. Nonlinear thermal radiation influence of Jeffrey nanofluid in three-dimensional flows is presented by Shehzad et al. [4]. Hussain and Ellahi [5] studied slip features of the flow of a Jeffrey fluid. [6] Hayyat et al. considered stagnation points flow in existence of Newtonian heating of Jeffrey nanofluid. Reddy et al. [7] studied Jeffrey fluid's flow as a result of oscillation of disks. Faroq et al. explored MHD flows of a Jeffrey fluid in occurrence of a Newtonian heating [8].

In contrast, an inclusive argument of a Jeffrey fluid on steady flows using stretching sheet can be originated in [9-14]. The analytical solution over a shrinking sheet of the Jeffrey fluid for boundary layer steady flow has been underlined by Nadeem et al. [15] whereas Hamad et al. [16] suggested a finite difference study to analyze the thermal jump effects near a stagnation points on the shrinking sheet using variable thermal conductivity on boundary layer flow of a Jeffrey fluid. References [17-29] are delivered for several thought-provoking outcomes of a Jeffrey fluid peristaltic flow. 
For the Jeffrey fluid, a more wide-ranging solution can be obtained when a fluid transpiration is considered on the wall. The leading equations of motion for Jeffrey fluid with wall transpiration are far complex as related with Jeffrey fluid without wall transpiration. This complexity of governing equations of Jeffrey fluid with wall transpiration creates the inconvenience to attain the exact solution, and it is challenging to handle. In the study of wall transpiration, most of the concern is by reason of its substantial uses in aeronautical system, controlled boundary layer, mechanical and chemical engineering processes, and for instance manufacturing techniques. Cruz and Lins [30] are associated to a recent published article on this line, which examines the unsteady flow of Newtonian fluid for Stokes' first and second problems, bounded by plate with wall transpiration. In their research, an analytical solution is presented for the fluid's behavior over the flat plates with oscillating and impulsive motions, beginning from rest and using wall transpiration.

This paper is extended by Hameed et al. [31]; they derived exact solution for unsteady flow of a second-grade fluid above flat plates with oscillating and impulsive motions, initially at rest, and by means of wall transpiration by Laplace transform, perturbation method, and an extended variable separation technique.

Stokes problems have countless importance in abundant fields of life, which contain the industry manufacturing, geophysical flows, chemical engineering, and a heat conduction phenomenon. Initially, in 1851, Stokes [32] presented a famous research study on pendulum in which the problems related to the oscillatory and impulsive motions of a plane were considered. Initially, Stokes' problems are boundary value one-dimensional problems to a viscous flows inspired by a moving plane. After simplified Navier-Stokes equations, the resulted partial differential equation is momentum equation that contains differentiation with respect to (w.r.t) two variables only, the coordinates which is normal to the time and plane. This work assists that the viscous flows of fluid are mathematically solvable. The related solution for both steady and transient state has been well deliberated [33-38].

The aim of this paper is to study the transient incompressible flows of Jeffrey fluids over a permeable, flat, and infinite plate. The plate motion is an oscillatory translation along the $x$-axis. Using the Laplace transform and perturbation method, the analytical solution for the nondimensional velocity field in the transform domain has been obtained. The velocity field in the real domain has been determined by using the numerical Stehfest's algorithm for the Laplace transform inversion. The particular case of the flow without transpiration is also investigated, and the analytical solution of the velocity field is determined. To have a validation of the obtained solution by the perturbation method, we have compared the analytical solution for the flow with transpiration with the solution corresponding to the flow without transpiration. It was found that for the transpiration parameter tending to zero, the solution for the flow with transpiration tends to the solution corresponding to the case without perspiration. The influence of the system parameters on fluid motion has been investigated by numerical simulations and graphical illustrations prepared with the software package Mathcad.

\section{Mathematical Modeling of the Problem}

Let us consider incompressible unsteady flows of a Jeffrey fluid over a flat porous plate situated in the $(x, z)$-plane of a Cartesian coordinate system $O x y z$; therefore, the $y$-axis is perpendicular to the plate (Figure 1). Initially, at $t=0$, the plate and fluid are at the rest. In this paper, we seek a velocity field of the form $\vec{V}(y, t)=u(y, t) \vec{e}_{x}+v(y, t) \vec{e}_{y}$, where $\vec{e}_{x}$ and $\vec{e}_{y}$ are the unit vectors in the $x$-and $y$-direction, respectively. The incompressibility condition implies that the velocity component $v(y, t)$ has to satisfy condition $\partial v(y, t) / \partial y=0$, so $v(y, t)=V(t)$. In this paper, for velocity component $v(y, t)$ to be constant, therefore, hereafter we consider $v(y, t)=-V_{0}$. After the instant $t=0$, the plate translates along $x$-axis with velocity $u(0, t)=U_{0} \exp (i \omega t)$, where $\omega$ is the oscillations frequency.

Under the above consideration, the governing equation for the flow of unsteady incompressible Jeffrey fluid over a porous plate has the following form $[3,11]$ :

$$
\rho\left(\frac{\partial u}{\partial t}-V_{0} \frac{\partial u}{\partial y}\right)=\frac{\mu}{1+\lambda_{1}}\left(\frac{\partial^{2} u}{\partial y^{2}}+\lambda_{2} \frac{\partial^{3} u}{\partial y^{2} \partial t}+\lambda_{2} V_{0} \frac{\partial^{3} u}{\partial y^{3}}\right), \quad y>0, t>0
$$

where $\mu$ denotes the dynamic viscosity of the fluid, $\rho$ represents the constant fluid's density, $V_{0}$ symbolizes the uniform transpiration (suction for $V_{0}>0$, respectively, blowing for $\left.V_{0}<0\right), \lambda_{1}$ represents the ratio of relaxation time to the retardation time, whereas $\lambda_{2}$ is the retardation time. It is noteworthy that by particularizing values of the parameters $\lambda_{1}$ and $\lambda_{2}$, the governing equations of Newtonian and secondgrade fluids can be obtained.

Along with equation (1), the following initial and boundary conditions are considered:

$$
\begin{aligned}
& u(y, 0)=0, \quad y \geq 0, \\
& u(0, t)=u_{0}(t)=U_{0} e^{i \omega t}, \quad t>0, \\
& u(y, t)=0 \text { as } y \longrightarrow \infty, \quad t \geq 0 .
\end{aligned}
$$

Now, to obtain the dimensionless form of the abovementioned equations, we use the set of dimensionless variables as follows: 


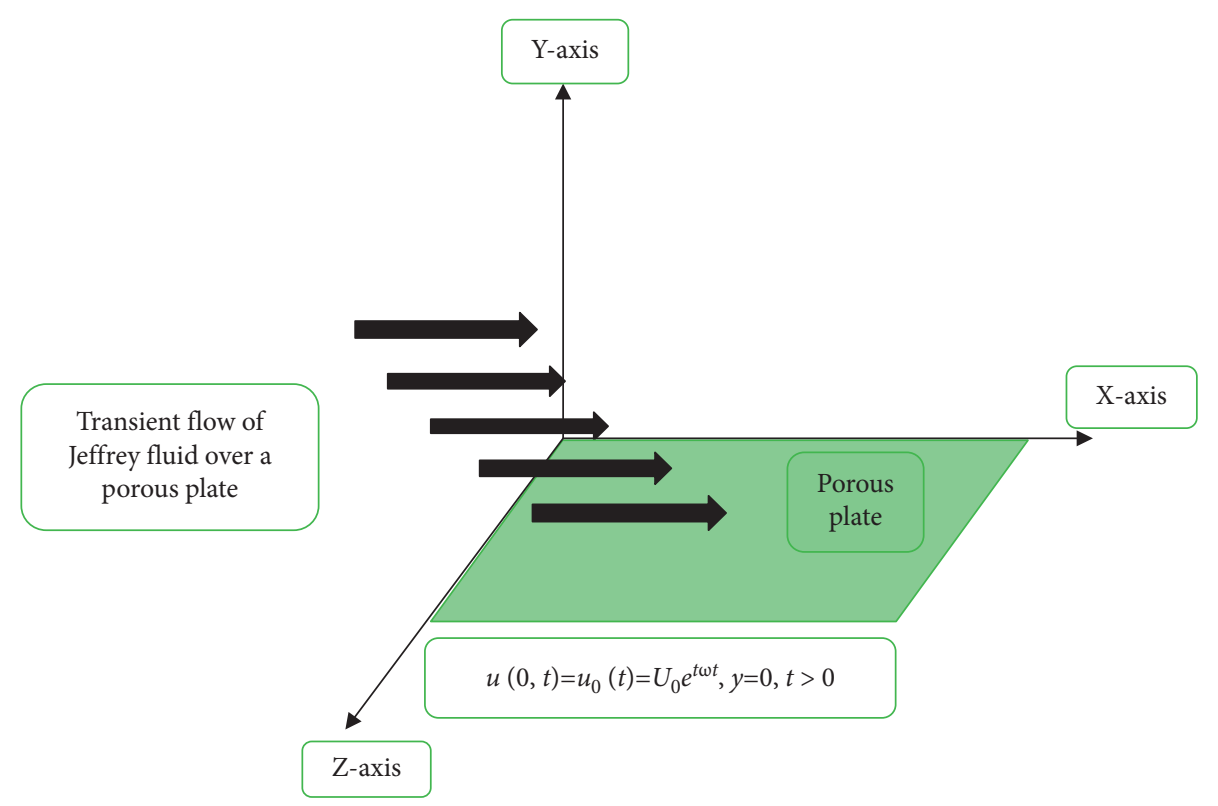

FIgURE 1: Geometry of the problem.

$$
\begin{aligned}
u^{*} & =\frac{u}{U_{0}}, \\
y^{*} & =\frac{U_{0} y}{v}, \\
t^{*} & =\frac{U_{0}^{2} t}{\nu}, \\
\omega^{*} & =\frac{\omega \nu}{U_{0}^{2}}, \\
\beta & =\frac{U_{0}^{2}}{\nu} \lambda_{2}, \\
2 \gamma & =\frac{V_{0}}{U_{0}},
\end{aligned}
$$

into equations (1)-(4). The dimensionless initial-boundary value problem (dropping the $*$ notation) is

$$
\begin{array}{r}
\frac{1}{1+\lambda_{1}}\left(\frac{\partial^{2} u}{\partial y^{2}}+\beta \frac{\partial^{3} u}{\partial y^{2} \partial t}+2 \beta \gamma \frac{\partial^{3} u}{\partial y^{3}}\right)+2 \gamma \frac{\partial u}{\partial y}-\frac{\partial u}{\partial t}=0, \\
u(y, 0)=0, \quad y \geq 0, \\
u(0, t)=u_{0}(t)=e^{i \omega t}, \quad t>0, \\
u(y, t)=0 \text { as } y \longrightarrow \infty, \quad t \geq 0,
\end{array}
$$

where $\beta$ is the Jaffrey fluid parameter and $\gamma$ represents wall transpiration parameter $(\gamma>0$ for suction and $\gamma<0$ for injection).

\section{Solution of the Problem}

To determine analytical solution of the problem formulated in Section 2, we use the Laplace transform with respect to time $t$ and the regular perturbation method. Applying the Laplace transform to equations (6), (8), and (9) and using the initial condition (7), the following problem in the transformed domain is obtained:

$$
\begin{aligned}
& \frac{1}{1+\lambda_{1}}(1+\beta s) \frac{\partial^{2} \bar{u}}{\partial y^{2}}+\frac{2 \beta \gamma}{1+\lambda_{1}} \frac{\partial^{3} \bar{u}}{\partial y^{3}}+2 \gamma \frac{\partial \bar{u}}{\partial y}-s \bar{u}=0, \\
& \bar{u}(0, s)=\frac{1}{s-\iota \omega}, \bar{u}(y, s)=0, \quad \text { as } y \longrightarrow \infty, t \geq 0,
\end{aligned}
$$

where $\bar{u}(y, s)=\int_{0}^{\infty} u(y, t) \exp (-s t) \mathrm{d} t$ is the Laplace transform of function $u(y, t)$ and $s$ is known as the transform parameter.

3.1. The Perturbed Analytical Solution. To solve equation (10) along with boundary conditions (11), we suppose that the Jeffrey parameter $\beta$ has small values; therefore, we suppose that $\beta \ll 1$ and the transformed velocity field $\bar{u}(y, s)$ is expressed as

$$
\bar{u}(y, s)=\bar{u}_{0}(y, s)+\beta \bar{u}_{1}(y, s)+0\left(\beta^{2}\right) .
$$

Now, substituting equation (12) into equations (10) and (11) and then comparing the powers of $\beta$, we obtain the following problems:

(i) The zero order problem: 


$$
\begin{aligned}
\bar{u}_{0}^{\prime \prime}+2 \gamma\left(1+\lambda_{1}\right) \bar{u}_{0}^{\prime}-\left(1+\lambda_{1}\right) s \bar{u}_{0} & =0, \\
u_{0}(0, s) & =\frac{1}{s-i \omega}, \\
u(\infty, s) & =0 .
\end{aligned}
$$

(ii) The first order problem:

$$
\begin{array}{r}
\bar{u}_{1}^{\prime \prime}+2 \gamma\left(1+\lambda_{1}\right) \bar{u}_{1}^{\prime}-\left(1+\lambda_{1}\right) s \bar{u}_{1}=-\left(2 \gamma \bar{u}_{0}^{\prime \prime \prime}+s \bar{u}_{0}^{\prime \prime \prime}\right), \\
u_{1}(0, s)=0, u_{1}(\infty, s)=0 .
\end{array}
$$

The solutions to the above equations are given by

$$
\begin{aligned}
& \bar{u}_{0}(y, s)=\frac{1}{s-\iota \omega} e^{-y \varphi(s)}, \\
& \bar{u}_{1}(y, s)=A(s) y e^{-y \varphi(s)},
\end{aligned}
$$

where

$$
\begin{aligned}
A(s) & =\frac{-\varphi^{2}(s)(2 \gamma \varphi(s)-s)}{2 \delta_{1} \sqrt{1+s / \delta_{1} \gamma}(s-i \omega)}, \\
\varphi(s) & =\delta_{1}\left(1+\sqrt{1+\frac{s}{\delta_{1} \gamma}}\right), \\
\delta_{1} & =\gamma\left(1+\lambda_{1}\right) .
\end{aligned}
$$

The transformed velocity field is given by

$$
\bar{u}(y, s)=\frac{1}{s-\iota \omega}\left[1+\frac{\beta y\left(s-2 \gamma \varphi(s) \varphi^{2}(s)\right)}{2 \delta_{1} \sqrt{1+s / \delta_{1} \gamma}}\right] e^{-y \varphi(s)} .
$$

The inverse Laplace of expression (17) is difficult to determine. For this reason, the numerical values of the velocity field in the real domain will be obtained using Stehfest's numerical algorithm. According to this algorithm, the numerical values of the function $u(y, t)$ are approximated by the following expression [37]:

$$
\begin{aligned}
u(y, t) & \cong \frac{\ln (2)}{t} \sum_{k=1}^{P} Q_{k} \bar{u}\left(y, \frac{k \ln (2)}{t}\right), \\
Q_{k} & =(-1)^{k+(P / 2)} \sum_{j=[k+1 / 2]}^{\min (k,(P / 2))} \frac{j^{(P / 2)}(2 j) !}{((P / 2)-j) ! j !(j-1) !(k-j) !(2 j-k) !} .
\end{aligned}
$$

3.2. Analytical Solution of the Problem without Transpiration $(\gamma=0)$. In this case, equation (6) becomes

$$
\left(1+\beta \frac{\partial}{\partial t}\right) \frac{\partial^{2} u(y, t)}{\partial y^{2}}=\left(1+\lambda_{1}\right) \frac{\partial u(y, t)}{\partial t} .
$$

Applying the Laplace transform to equation (19) and using the initial condition (7), we obtain the transformed equation as follows:

$$
\frac{\partial^{2} \bar{u}(y, s)}{\partial y^{2}}=\frac{\left(1+\lambda_{1}\right) s}{(1+\beta s)} \bar{u}(y, s)
$$

Function $\bar{u}(y, s)$ in (20) has to satisfy the boundary condition (11). The analytical solution of the problem described by equations (20) and (11) is given by

$$
\begin{aligned}
\bar{u}(y, s) & =\frac{1}{s-i \omega} \exp \left(-y \sqrt{\frac{a_{0} s}{s+b_{0}}}\right), \\
a_{0} & =\frac{1+\lambda_{1}}{\beta}, \\
b_{0} & =\frac{1}{\beta} .
\end{aligned}
$$

Transform (21) can be written in the equivalent form as follows:

$$
\begin{aligned}
& \bar{u}(y, s)=\left(1+\frac{i \omega}{s-i \omega}\right) F(y, s), \\
& F(y, s)=\frac{1}{s} \exp \left(-y \sqrt{\frac{a_{0} s}{s+b_{0}}}\right) .
\end{aligned}
$$

Using the following inversion formula:

$$
\begin{aligned}
f(y, t)=L^{-1}\{F(y, s)\} & =L^{-1}\left\{\frac{1}{s} \exp \left(-y \sqrt{\frac{a_{0} s}{s+b_{0}}}\right)\right\} \\
& =1-\frac{2 a_{0}}{\pi} \int_{0}^{\infty} \frac{\sin (y z)}{z\left(a_{0}+z^{2}\right)} \exp \left(\frac{-b_{0} z^{2} t}{a_{0}+z^{2}}\right) \mathrm{d} z,
\end{aligned}
$$

we obtain the velocity field given by

$$
\begin{aligned}
u(y, t) & =\left(\delta(t)+i \omega e^{i \omega t}\right) * f(y, t) \\
& =f(y, t)+i \omega \int_{0}^{t} e^{i \omega(t-\tau)} f(y, \tau) \mathrm{d} \tau
\end{aligned}
$$

where $\delta(t)$ is the Dirac distribution.

It is important to emphasize that solution (24) can be used to validate the solution given by (17) and (18) for the general case of the flow with transpiration on the wall. For this, we show in Figure 2 that for $\gamma \longrightarrow 0$, the graphs of solution (17) tend to overlap the graph of function (24). To draw the curves in Figure 2, we used the following values of 


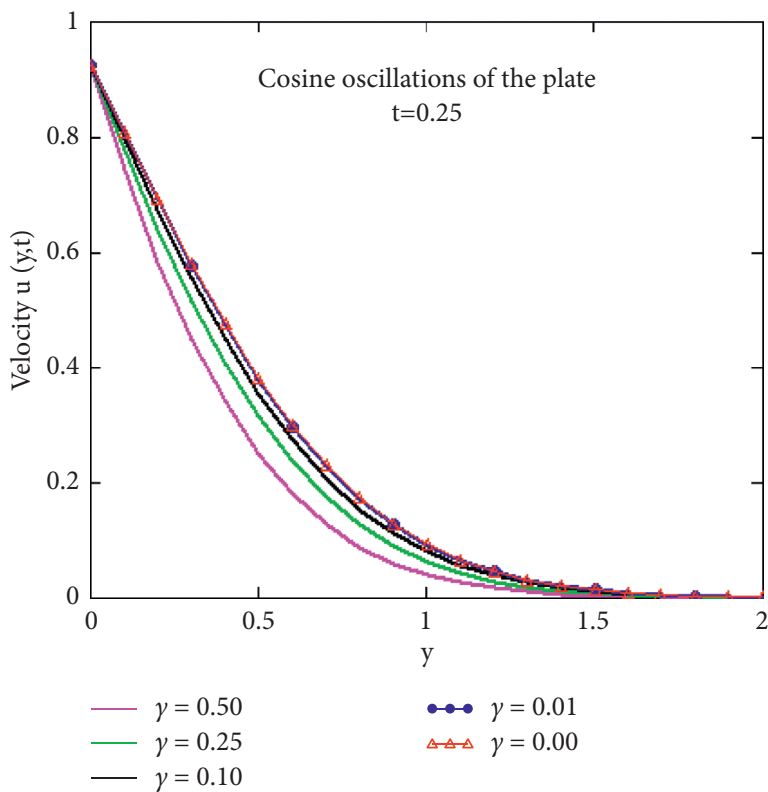

FIgURE 2: Comparison between solutions (17) and

the parameters: $\quad \lambda_{1}=0.45, \omega=\pi / 2, \beta=0.01, \quad$ and $\gamma \in\{0.50,0.25,0.01,0.00\}$. It is observed in Figure 2 that when the values of the transpiration parameter tend to zero, graphs of curves given by equation (1) tend to the graph of the solution given by (24). This shows that the perturbed solution of the problem is correct.

\section{Results and Discussion}

In the current work, approximate analytical solutions for the unsteady flows of a Jeffrey fluid over a porous plate have been obtained by using Laplace transform and perturbation techniques. The variant behavior of the velocity of Jeffrey fluid with different values of resulting parameters is displayed graphically in Figures $3-7$. These nondimensional emerging parameters are ratio of relaxation and retardation time $\lambda_{1}$, Jeffrey fluid parameter $\beta$, wall transpiration parameter $\gamma$, oscillating frequency $\omega$, and time $t$. The following figures show the velocity profiles for different values of dimensionless parameters of an unsteady flow of Jeffrey fluid above the porous plate.

The effect of ratio of relaxation and retardation time $\lambda_{1}$ on velocity distribution can be observed in Figure 3. We see that velocity decreased with the increasing values of $\lambda_{1}$. The fluid flow is therefore noticeably decelerated with an increase in relaxation time (or decrease in retardation time) because increment in $\lambda_{1}$ leads to increment in relaxation time; it means particles need much more time to come back from the perturbed system to equilibrium system in which subsequently fluid velocity decreases.

In Figure 4, we observe the effect of Jeffrey fluid parameter $\beta$ on velocity. It is observed that with the increment in Jeffrey fluid parameter, the velocity profile decreases. It is found that the momentum boundary layer thickness increases as the Jeffrey fluid parameter effect is strengthened. Hence, the velocity profile decreases due to this fact.

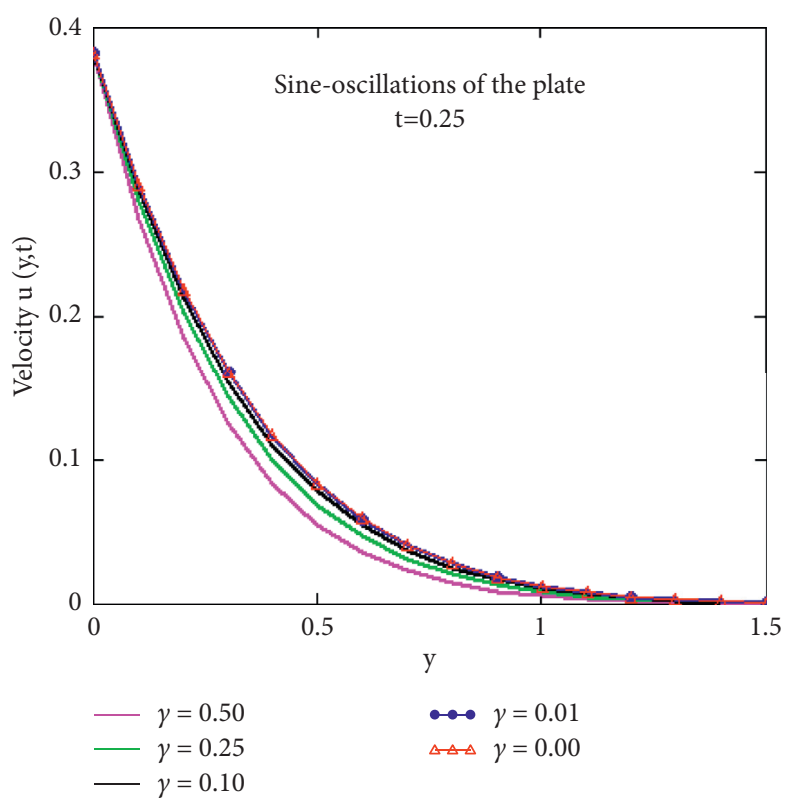

(24) for the transpiration parameter tending to zero.

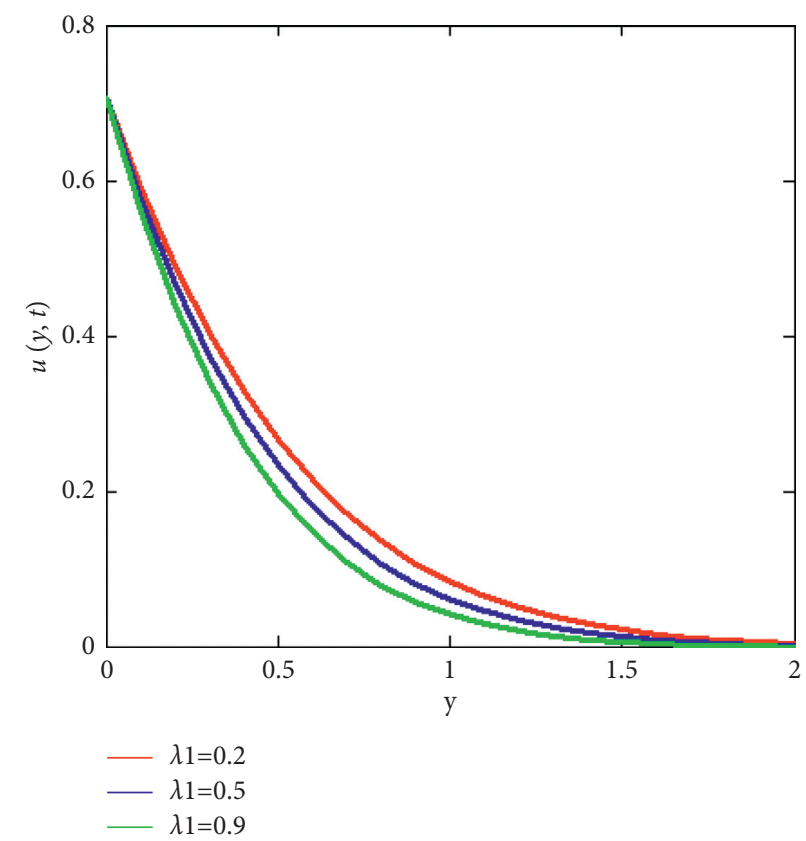

FIGURE 3: The influence of parameter $\lambda_{1}$ on velocity when $\beta=0.01, \omega=\pi / 2, \gamma=0.1$, and $t=0.5$.

The behavior of velocity under the effect of wall transpiration parameter $\gamma$ is observed with the help of Figure 5. It is perceived that the velocity of Jeffrey fluid flow is decreased along with increasing wall transpiration (injection/ suction parameter) parameter. In fact, fluid experiences greater viscosity with the porous boundaries and hence offers resistance to flow resulting reduction in the velocity. For large values of parameter, the decay is more.

In order to study the effect of frequency of oscillation $\omega$, we have plotted Figure 6, where it shows that the velocity of the Jeffrey fluid is also a strong function of $\omega$. The effect of 


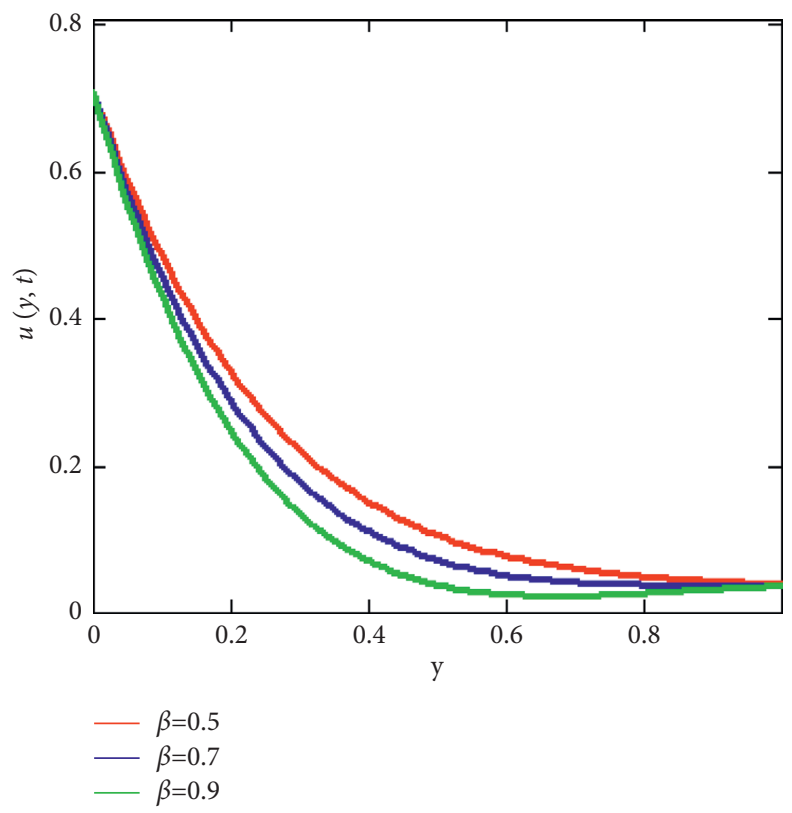

FIgURE 4: Influence of $\beta$ on velocity for $\lambda_{1}=0.2, \omega=\pi / 2, \gamma=0.1$, and $t=0.5$.

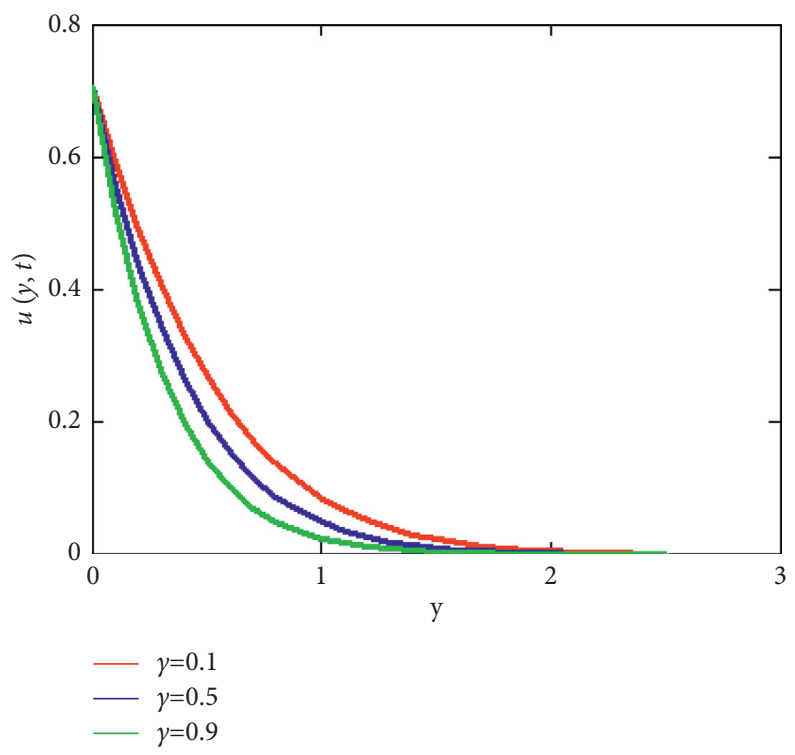

FIgURE 5: Influence of $\gamma$ on velocity when $\lambda_{1}=0.2, \omega=\pi / 2, \beta=0.01$, and $t=0.5$. 


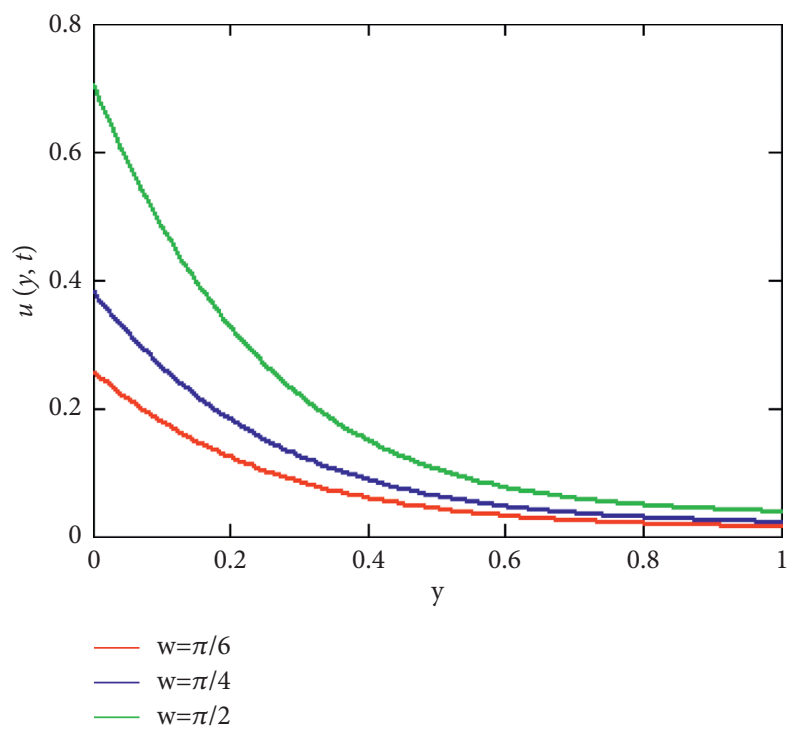

Figure 6: Influence of $\omega$ on velocity when $\lambda_{1}=0.2, \gamma=0.1, \beta=0.01$, and $t=0.5$.

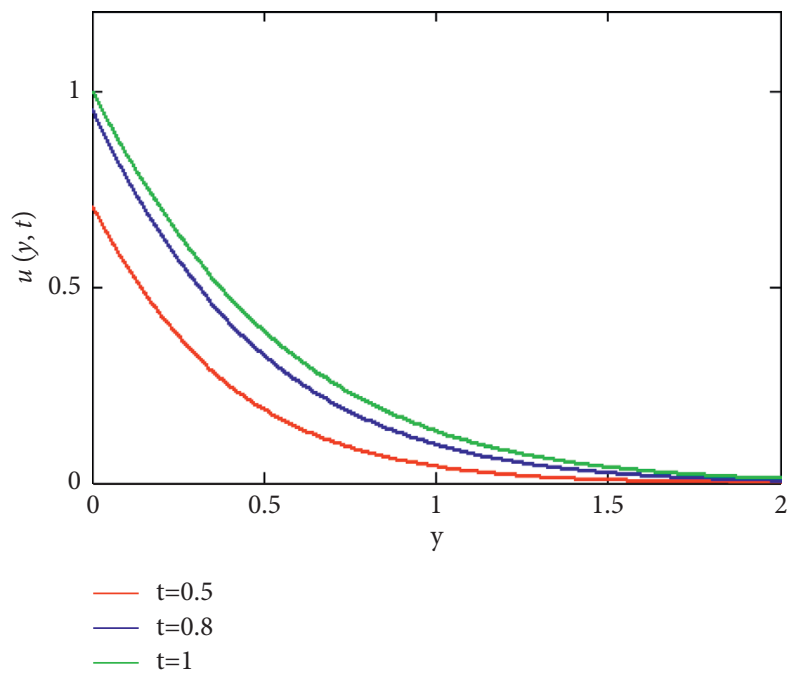

Figure 7: Influence of $(t)$ on velocity when $\lambda_{1}=0.2, \omega=\pi / 2, \beta=0.01$, and $\gamma=0.1$.

frequency of oscillation on the velocity profile is opposite as that of the ratio of relaxation to retardation time $\lambda_{1}$.

Figure 7 shows the relationship between velocity of the fluid and time. We see that the velocity enhances by increasing the value of dimensionless time $t$.

\section{Conclusion}

This work presents approximate analytical solutions for the unsteady flows of Jeffrey fluids over a porous, oscillating plate. Approximate analytical solutions for the nondimensional velocity field in the transformed domain have been obtained using the Laplace transform and perturbation method. The velocity field in the real domain has been determined by using the numerical Stehfest's algorithm for the Laplace transform inversion. The particular case of flow without transpiration has been studied. The comparison between both solutions, namely, the solution with transpiration when the transpiration parameter approaches zero and the solution without transpiration has shown that for the transpiration parameter tending to zero, the solution for the flow with transpiration tends to the solution corresponding to the case without perspiration. The influence of the system parameters on fluid motion has been investigated by numerical simulations and graphical illustrations prepared with the software package Mathcad.

\section{Data Availability}

No data were used to support this research.

\section{Conflicts of Interest}

The authors declare that they have no conflicts of interest. 


\section{References}

[1] D. Tripathi, N. Ali, T. Hayat, M. K. Chaube, and A. A. Hendi, "Peristaltic flow of MHD Jeffrey fluid through a finite length cylindrical tube," Applied Mathematics and Mechanics, vol. 32, pp. 1148-1160, 2011.

[2] K. Das, "Influence of slip and heat transfer on MHD peristaltic flow of a Jeffrey fluid in an inclined asymmetric porous channel," Indian Journal of Mathematics, vol. 54, pp. 19-45, 2012.

[3] M. A. A. Hamad, S. M. A. Gaied, and W. A. Khan, "Thermal jump effects on boundary layer flow of a Jeffrey fluid near the stagnation point on a stretching/shrinking sheet with variable thermal conductivity," Journal of Fluids, vol. 2013, Article ID 749271, , 2013.

[4] S. A. Shehzad, T. Hayat, A. Alsaedi, and M. A. Obid, "Nonlinear thermal radiation in three-dimensional flow of Jeffrey nanofluid: a model for solar energy," Applied Mathematics and Computation, vol. 248, pp. 273-286, 2014.

[5] R. Ellahi and F. Hussain, "Simultaneous effects of MHD and partial slip on peristaltic flow of Jeffery fluid in a rectangular duct," Journal of Magnetism and Magnetic Materials, vol. 393, pp. 284-292, 2015.

[6] T. Hayat, M. Imtiaz, and A. Alsaedi, "Magneto-hydrodynamic stagnation point flow of a Jeffrey nanofluid with Newtonian heating," Journal of Aerospace Engineering, vol. 29, no. 3, 2015.

[7] G. Bhaskar Reddy, S. Sreenadh, R. Hemadri Reddy, and A. Kavitha, "Flow of a Jeffrey fluid between torsionally oscillating disks," Ain Shams Engineering Journal, vol. 6, no. 1, pp. 355-362, 2015.

[8] M. Farooq, N. Gull, A. Alsaedi, and T. Hayat, "MHD flow of a Jeffrey fluid with Newtonian heating," Journal of Mechanics, vol. 33, pp. 1-11, 2015.

[9] N. Dalir, M. Dehsara, and S. S. Nourazar, "Entropy analysis for magnetohydrodynamic flow and heat transfer of a Jeffrey nanofluid over a stretching sheet," Energy, vol. 79, pp. 351362, 2015.

[10] M. Qasim, "Heat and mass transfer in a Jeffrey fluid over a stretching sheet with heat source/sink," Alexandria Engineering Journal, vol. 52, no. 4, pp. 571-575, 2013.

[11] T. Hayat, S. Asad, M. Mustafa, and A. Alsaedi, "MHD stagnation-point flow of Jeffrey fluid over a convectively heated stretching sheet," Computers \& Fluids, vol. 108, pp. 179-185, 2015.

[12] T. Hayat, M. Awais, and A. Alsaedi, "Newtonian heating and magnetohydrodynamic effects in flow of a Jeffrey fluid over a radially stretching surface," International Journal of the Physical Sciences, vol. 21, pp. 2838-2844, 2012.

[13] N. Dalir, "Numerical study of entropy generation for forced convection flow and heat transfer of a Jeffrey fluid over a stretching sheet," Alexandria Engineering Journal, vol. 53, no. 4, pp. 769-778, 2014.

[14] T. Hayat, M. Awais, and S. Obaidat, "Three-dimensional flow of a Jeffery fluid over a linearly stretching sheet," Communications in Nonlinear Science and Numerical Simulation, vol. 17, no. 2, pp. 699-707, 2012.

[15] S. Nadeem, A. Hussain, and M. K, "Stagnation flow of a Jeffrey fluid over a shrinking sheet," Zeitschrift für Naturforschung A, vol. 65, no. 6-7, pp. 540-548, 2010.

[16] M. A. A. Hamad, S. M. Abdel-Gaied, and W. A. Khan, "Thermal jumps effects on boundary layer flow of a Jeffrey fluid near the stagnation point on a stretching/shrinking sheet with variable thermal conductivity," Journal of Fluids, vol. 2013, Article ID 749271, , 2013.
[17] T. Hayat, N. Ahmad, and N. Ali, "Effects of an endoscope and magnetic field on the peristalsis involving Jeffrey fluid," Communications in Nonlinear Science and Numerical Simulation, vol. 13, no. 8, pp. 1581-1591, 2008.

[18] S. Nadeem and S. Akram, "Influence of inclined magnetic field on peristaltic flow of a Jeffrey fluid with heat and mass transfer in an inclined symmetric or asymmetric channel," Asia- $\mathrm{Pa}$ cific Journal of Chemical Engineering, vol. 7, no. 1, pp. 33-44, 2012.

[19] A. M. Abd-Alla and S. M. Abo-Dahab, "Magnetic field and rotation effects on peristaltic transport of a Jeffrey fluid in an asymmetric channel," Journal of Magnetism and Magnetic Materials, vol. 374, pp. 680-689, 2015.

[20] N. S. Akhbar, S. Nadeem, and C. Lee, "Characteristics of Jeffrey model for peristaltic flow of chyme in small intestine with magnetic field," Results in Physics, vol. 3, pp. 152-160, 2013.

[21] S. R. Mahmoud, "Effect of rotation and magnetic field through porous medium on peristaltic transport of a Jeffrey fluid in tube," Mathematical Problems in Engineering, vol. 2011, Article ID 971456, 13 pages, 2011.

[22] S. Akram and S. Nadeem, "Influence of induced magnetic field and heat transfer on the peristaltic motion of a Jeffrey fluid in an asymmetric channel: closed form solutions," Journal of Magnetism and Magnetic Materials, vol. 328, pp. 11-20, 2013.

[23] N. S. Akhbar and S. Nadeem, "Mixed convective magnetohydrodynamics peristaltic flow of a Jeffrey nano-fluid with Newtonian heating," Zeitschrift für Naturforschung A, vol. 68, pp. 433-441, 2013

[24] T. Hayat and N. Ali, "Peristaltic motion of a Jeffrey fluid under the effect of a magnetic field in a tube," Communications in Nonlinear Science and Numerical Simulation, vol. 13, no. 7, pp. 1343-1352, 2008.

[25] A. Afsar Khan, R. Ellahi, and K. Vafai, "Peristaltic transport of a Jeffrey fluid with variable viscosity through a porous medium in an asymmetric channel," Advances in Mathematical Physics, vol. 2012, Article ID 169642, 15 pages, 2012.

[26] A. Ebaid, "Remarks on the homotopy perturbation method for the peristaltic flow of Jeffrey fluid with nano-particles in an asymmetric channel," Computers \& Mathematics with Applications, vol. 68, no. 3, pp. 77-85, 2014.

[27] S. Nadeem and S. Akram, "Slip effects on the peristaltic flow of a Jeffrey fluid in an asymmetric channel under the effect of induced magnetic field," International Journal for Numerical Methods in Fluids, vol. 63, no. 3, pp. 374-394, 2010.

[28] K. Vajravelu, S. Sreenadh, and P. Lakshminarayana, "The influence of heat transfer on peristaltic transport of a Jeffrey fluid in a vertical porous stratum," Communications in Nonlinear Science and Numerical Simulation, vol. 16, no. 8, pp. 3107-3125, 2011.

[29] N. S. Akhbar and S. Nadeem, "Thermal and velocity slip effects on the peristaltic flow of a 6-constant Jeffrey's fluid model," International Journal of Heat and Mass Transfer, vol. 55, pp. 364-3970, 2012.

[30] D. O. De Almeida Cruz and E. Ferreira Lins, "The unsteady flow generated by an oscillating wall with transpiration," International Journal of Non-Linear Mechanics, vol. 45, no. 4, pp. 453-457, 2010.

[31] M. Abdulhameed, I. Khan, D. Vieru, and S. Shafie, "Exact solutions for unsteady flow of second grade fluid generated by oscillating wall with transpiration," Applied Mathematics and Mechanics, vol. 35, no. 7, pp. 821-830, 2014. 
[32] G. G. Stokes, "On the effect of the internal friction of fluids on the motion of pendulums," Transaction of the Cambridge Philosophical Society, vol. 9, pp. 8-106, 1851.

[33] M. E. Erdogan, "A note on an unsteady flow of a viscous fluid due to an oscillating plane wall," International Journal of NonLinear Mechanics, vol. 35, no. 1, pp. 1-6, 2000.

[34] C.-M. Liu and I.-C. Liu, "A note on the transient solution of Stokes' second problem with arbitrary initial phase," Journal of Mechanics, vol. 22, no. 4, pp. 349-354, 2006.

[35] R. Panton, "The transient for Stokes's oscillating plate: a solution in terms of tabulated functions," Journal of Fluid Mechanics, vol. 31, no. 4, pp. 819-825, 1968.

[36] H. Schlichting, Boundary Layer Theory, McGraw-Hill, New York, NY, USA, 1979.

[37] A. Ara, N. A. Khan, F. Sultan, and S. Ullah, "Numerical simulation of Jeffery-Hamel flow of Bingham Plastic fluid and heat transfer in the presence of magnetic field," Applied and Computational Mathematics, vol. 18, no. 2, pp. 135-148, 2019.

[38] B. Davies and B. Martin, "Numerical inversion of the Laplace transform: a survey and comparison of methods," Journal of Computational Physics, vol. 33, no. 1, pp. 1-32, 1979. 